\title{
Development to enable precision medicine in Africa
}

\author{
Nicola Mulder*,1 \\ ${ }^{1}$ Computational Biology Division, Department of Integrative Biomedical Sciences, IDM, University of Cape Town, Cape Town, \\ South Africa \\ * Author for correspondence: nicola.mulder@uct.ac.za
}

"While extensive research has been carried out on these diseases in European populations, there is a dearth of data and research on African populations."

First draft submitted: 26 July 2017; Accepted for publication: 10 August 2017; Published online: 24 November 2017

Keywords: Africa • genetic variant • genomic medicine $\bullet$ translational medicine

Precision medicine enables the customization of patient care driven by experimental data and knowledge about an individual or a population. Though the data can include omics data from different technologies, this article focuses on the use of genomic data for improving the precision of diagnosis and treatment. Precision medicine in developed countries is enabled by robust research and health infrastructures, data analysts and health care professions skilled in genomic medicine and availability of reference datasets from control and disease cohorts. Extensive literature on the genetic and environmental associations with diseases is available for 'Western' populations, providing an excellent platform for the development of new diagnostics (e.g., genetic tests), data driven choice of and dosing for therapeutics and thus effective implementation of precision medicine. In contrast, Africa carries the highest burden of both infectious and noncommunicable diseases on earth and at the same time, hosts the greatest genetic diversity within its populations [1]. Tuberculosis, HIV and malaria dominate both in number of cases and research focus for infectious diseases, but there is increasing interest in the high burden of noncommunicable diseases such as diabetes and cardiovascular diseases, among others. While extensive research has been carried out on these diseases in European populations, there is a dearth of data and research on African populations. A recent analysis of data in the Genome Wide Association Study Catalog determined that though the proportion has increased in recent years, less than $10 \%$ of the data comes from African populations [2]. There is also disparity in the research outputs of African scientists; however, an analysis from 2015 has shown that we are seeing an increase in the number of research articles led by African scientists, along with an increase in publications from Africa related to health research [3].

\section{Data for the African context}

For precision medicine to become a reality in Africa we need more research and more data that is applicable to indigenous populations. Using data from non-African populations for diagnosis could result in African individuals mistakenly considered to have a disease risk variant [2]; if more extensive data was available for African populations, these variants may turn out to be reasonably common in these populations. There are some well-known disease causing variants that appear to be over-represented in African populations and over the last decade, new disease risk associated variants have been discovered in these populations. Researchers have also discovered a number of cases where disease associated or pharmacogenomic variants identified in European populations have significantly different frequencies and thus potential impact in African populations. These can also differ substantially between different African populations. For example, Koshy et al. [4] found notable interethnic differences in the allele frequencies of single nucleotide variants (SNVs) in TGM1 which is associated with autosomal recessive lamellar ichthyosis. A notable example of an 'African' genetic disease is sickle cell disease (SCD), for which $64 \%$ of new born cases annually are from sub-Saharan Africa [5]. SCD is caused by an SNV in the $\beta$-globin gene which results in a Glu6Val substitution. This causes polymerization of hemoglobin S, resulting in erythrocytes with a sickle shape [6].

Future 8 Medicine 
SCD is associated with many cardiovascular-related and other diseases, with different severities, depending on a number of factors, including additional SNVs. A few studies have been done on the association between genetic determinants and phenotype severity, but not in all African populations. More genomics research is needed to better understand the link between genetics and different manifestations of SCD-associated diseases in different African populations [7]. There are also few studies on attempting to understand the link between genetics and variable responses to pain in SCD [6].

Pharmacogenomics in African populations is another under-represented area of research, but examples to date have demonstrated the importance of using data for the population being treated. For SCD, for example, some SNV s have been identified in the SAR1, BCL11A and HBS1L-MYB genes that are associated with altered response to hydroxyurea treatment [6]. Efficacy of therapeutics for the key infectious diseases, TB and HIV, has also been found to be impacted by genetics. The SLCO1B1 gene, which encodes a membrane-bound organic anion transporter protein, is involved in transport of compounds and SNVs in this gene are associated with variations in response to rifampicin, an essential therapy for TB. Aklillu et al. [8] have shown that the allele frequencies in SLCO1B1 differ significantly between African and European populations and also demonstrate inter-population differences, suggesting the need for more detailed studies in different African populations in order to implement more precise dosing. For HIV, efavirenz (EFV) is the preferred first-line antiretroviral therapy, but has recently been associated with neuropsychiatric adverse effects. Genetic variants in $C Y P 2 B 6$, which have been found at higher frequency in several African populations, affect the metabolism of EFV, thus predisposing these populations to the adverse effects. This argues for pharmacogenetics testing of $C Y P 2 B 6$ to improve the precision of EFV dosing [9].

\section{Building infrastructure \& resources}

These examples, among many others, demonstrate the need to exploit the diversity across different populations and diseases in Africa to prepare for precision medicine on the continent. Preparation for the implementation of precision medicine requires several resources to be in place, including access to reference population data, extensive research on specific diseases in individual populations, skills in genomic data analysis and interpretation and basic research and clinical infrastructure for carrying out research and routine genetics testing. Currently, in most African institutions, particularly from low income countries, research infrastructure is poor and the number of trained scientists is inadequate. However, there are several examples of successful research networks that have been developed in the health sector in Africa that are addressing these challenges, including the Wellcome Trust DELTAS program, the GSK Africa OpenLab and the Human Heredity and Health in Africa (H3Africa) initiative [10]. These initiatives have been very successful in driving African-led science, building infrastructure and developing a vibrant, productive scientific community. H3ABioNet, the pan-African bioinformatics network of H3Africa [11], has been instrumental in developing skills and infrastructure for genomics data analysis and together with training programs of the individual H3Africa projects and other initiatives, is developing a formidable workforce across the spectrum of genomics research. H3Africa has developed research infrastructure in many African institutions, developed new training modalities and built a supportive network of scientists. Its working groups have collectively addressed issues of ethics consent for data sharing, study coordination, sample shipping, data generation and data processing, among other challenges facing African scientists.

Resources being developed by H3Africa and other large-scale genomics projects on the continent are generating data for over 80,000 participants in Africa and cover multiple diseases. These constitute an excellent base from which to build a large-sale African cohort, providing both reference and disease data. However, given the diversity of populations and diseases, even more data is required. Sequencing of whole genomes or exomes, remains expensive for African researchers, but enables the discovery of rare variants if large sample sizes are available. For common variants, a more feasible alternative is genotyping arrays which are cheaper to run and analyze [2]. The H3Africa consortium has designed a new genotyping array, which is more applicable to African populations as the design is based on the analysis of whole genome sequence data from more than 3000 African individuals from diverse populations. This 2.5 million marker chip will be used for over 30,000 samples in the first instance, which will create a valuable dataset for disease variant discovery and reference population data. The array design has included the development of an African reference panel for imputation, which could be further improved by including additional sequence data from more diverse populations. Ideally we need an 'African Genome Project' to increase the reference sequence data. Of course the next challenge is having the skills and computing infrastructure to analyze these large datasets. We need to train bioinformaticians, as well as to transfer genomic medicine skills to clinical research scientists and healthcare professionals. We also need research infrastructure including research and computing equipment. To 
address this, $\mathrm{H} 3 \mathrm{ABioNet}$ has developed and is monitoring usage of computing infrastructure at various institutions across the continent. As mentioned previously, the network also has an extensive training program for equipping bioinformaticians, researchers and health care professionals with skills in data analysis and interpretation, but is also training systems administrators and software developers to maintain the computing infrastructure and develop reproducible data analysis workflows.

\section{Data harmonization \& access}

In addition to genomic data, several projects are collecting rich phenotype data and efforts are being made to standardize and harmonize the data. Across the H3Africa consortium, for example, a Phenotype Harmonization Working Group and Ontologies Task Force has established a set of core phenotypes that should be collected by all projects and developed recommendations for how these data are collected in case report forms to ensure it is comparable. Also within the consortium is a Cardiovascular Disease Working Group comprising members of projects studying the genetic determinants of cardiovascular-related diseases, which is making a concerted effort to provide harmonized clinical data for over 50,000 individuals [12].

To ensure usefulness of research efforts in genomic medicine, an essential outcome must be the accessibility of the phenotype and genomic data. For H3Africa, the data is required to be deposited in public repositories such as the European Genome-phenome archive, with access controlled by a Data and Biospecimen Access Committee. However, even more useful would be a browser where individual variants and their frequencies could be retrieved, as many researchers do not want to and often are not equipped with resources to download and analyze multiple datasets for a handful of SNVs of interest to them. Such an effort has been made for Middle East and North African data, where over 26 million genetic variations from multiple datasets have been annotated and their allele frequencies computed and made available via a web interface (known as al mena) [4]. Developing a European Variant Archive-like browser for all African variants would have enormous value for progressing research and facilitating clinical applications of genomic data. However there are many issues associated with ethics, privacy and consent which need to be addressed before this becomes a reality. The Global Alliance for Genomics and Health [13] (see the website [14]) is addressing some of these challenges and developing technical solutions for the secure and responsible sharing of genomic data to progress scientific discovery in the health sector.

\section{Translating research}

Finally, to make precision medicine a reality, we need to convert genomics knowledge into diagnostics, genetic tests or improved dosing algorithms. Since genomic medicine assumes disease susceptibility, severity or response to treatment is associated with genetic variation, gene expression or epigenetics, genetic testing is essential for multiple diseases, including single gene disorders with a Mendelian inheritance pattern as well as complex multifactorial diseases [15]. Scientists should partner with pharmaceutical companies for translational medicine and need to solicit commitment from their governments to establish facilities for enabling translational research and routine genetic testing and interpretation. Though sequencing is still an expensive and thus an exclusive option, with the know-how developed and data generated by the large-scale genomics projects, cost-effective custom arrays or genetic tests can be developed for newborn screening, pharmacogenomics or other diagnostic tools, which are appropriate for African populations. Whatever the solution, healthcare professionals need to work alongside data analysts to streamline the process.

Meeting the challenge of building a sustainable research and translational enterprise for precision medicine in Africa will, in the first instance require a close partnership between African and non-African sources of support. There are also benefits to collaborative development of such infrastructures, particularly in resource limited settings, where resources and efforts should be pooled to create critical mass. If these challenges are met, an African Precision Medicine Initiative at the population level is not an unfeasible proposition in the current generation of genomic medicine, particularly with the decreasing costs of data generation. If we have data and tools to intervene early in treatment of patients we can reduce the cost of treatment and care. African resources are also highly applicable to other continents who have to treat indigenous and immigrant populations of African descent, a perfect example being the large African-American community in the USA.

\section{Disclaimer}

The content in this article is solely the responsibility of the author and does not necessarily represent the official views of the National Institutes of Health. 
Financial \& competing interests disclosure

The H3ABioNet project, which is part of the H3Africa consortium and discussed in the paper, is funded by the NIH, see statement below. N Mulder is the PI of the H3ABioNet project. Research reported in this publication was supported by National Human Genome Research Institute (NHGRI) and the Office Of The Director (OD), National Institutes of Health under award number U41HG006941. The author has no other relevant affiliations or financial involvement with any organization or entity with a financial interest in or financial conflict with the subject matter or materials discussed in the manuscript apart from those disclosed.

No writing assistance was utilized in the production of this manuscript

\section{Ethical conduct of research}

The author states that she has obtained appropriate institutional review board approval or has followed the principles outlined in the Declaration of Helsinki for all human or animal experimental investigations. In addition, for investigations involving human subjects, informed consent has been obtained from the participants involved.

\section{Open access}

This work is licensed under the Attribution-NonCommercial-NoDerivatives 4.0 Unported License. To view a copy of this license, visit http://creativecommons.org/licenses/by-nc-nd/4.0/

\section{References}

1. Schlebusch CM, Skoglund P, Sjödin P et al. Genomic variation in seven khoe-san groups reveals adaptation and complex african history. Science 338(6105), 374-379 (2012).

2. Popejoy AB, Fullerton SM. Genomics is failing on diversity. Nature 538, 161-164 (2016).

3. Uthman OA, Wiysonge CS, Ota MO et al. Increasing the value of health research in the WHO African region beyond 2015 - reflecting on the past, celebrating the present and building the future: a bibliometric analysis. BMJ Open 5(3), e006340 (2016).

4. Koshy R, Ranawat A, Scaria V. al mena: a comprehensive resource of human genetic variants integrating genomes and exomes from Arab, Middle Eastern and North African populations. J. Hum. Genet. 62(10), 889-894 (2017).

5. Makani J, Ofori-Acquah SF, Tluway F, Mulder N, Wonkam A. Sickle cell disease: tipping the balance of genomic research to catalyse discoveries in Africa. Lancet 389(10087), 2355-2358 (2017).

6. Mnika K, Pule GD, Dandara C, Wonkam A. An expert review of pharmacogenomics of sickle cell disease therapeutics: not yet ready for global precision medicine. OMICS 20(10), 565-574 (2016).

7. Geard A, Pule GD, Chelo D, Bitoungui VJ, Wonkam A. Genetics of sickle cell-associated cardiovascular disease: an expert review with lessons learned in Africa. OMICS 20(10), 581-592 (2016).

8. Aklillu E, Habtewold A, Ngaimisi E et al. SLCO1B1 gene variations among Tanzanians, Ethiopians, and Europeans: relevance for African and worldwide precision medicine. OMICS 20(9), 538-545 (2016).

9. Masimirembwa C, Dandara C, Leutscher PD. Rolling out efavirenz for HIV precision medicine in Africa: are we ready for pharmacovigilance and tackling neuropsychiatric adverse effects? OMICS 20(10), 575-580 (2016).

10. The H3Africa Consortium. Enabling African scientists to engage fully in the genomic revolution. Science 344(6190), 1346-1348 (2014).

11. Mulder NJ, Adebiyi E, Alami R et al. H3ABioNet, a sustainable pan African bioinformatics network for human heredity and health in Africa. Genome Res. 26, 271-277 (2015).

12. Peprah E, Wiley K, Troyer J et al. Building a platform to enable NCD research to address population health in Africa: CVD working group discussion at the sixth H3Africa consortium meeting in Zambia. Glob. Heart 11(1), 165-170 (2016).

13. The Global Alliance for Genomics and Health. Page A, Baker D, Bobrow M et al. Enabling a federated data ecosystem for sharing genomic and clinical information. Science 352(6291), 1278-1280 (2016).

14. Global Alliance for Genomics and Health. http://genomicsandhealth.org/

15. Kotze MJ, Lückhoff HK, Peeters AV et al. Genomic medicine and risk prediction across the disease spectrum. Crit. Rev. Clin. Lab. Sci. 52(3), 120-137 (2015). 